\title{
New Robotic Technologies for Inspecting Two Pole Electric Generators while the Rotor Remains in Place
}

\author{
Mark Savenkov, Michael Turner \\ Alstom Power, Thermal Services Asia \\ Email: mark.savenkov@power.alstom.com
}

Received January 2015

\begin{abstract}
The electric generator is a highly stressed plant component requiring periodic inspection and maintenance to reduce the risk of a costly forced outage. This paper briefly introduces two new robotic technologies for performing fast and reliable inspections of two pole electric generators with minimal mechanical disassembly requirements. The first robotic system is designed to inspect within the generator rotor and stator air gap, while the second robotic system is designed to inspect the generator retaining rings. An overview of the design and construction of each system is provided, along with an explanation of the capabilities and benefits they bring to the power station owner.
\end{abstract}

Keywords

Generator, Condition Based Maintenance, Robotic Inspection, Retaining Rings

\section{Introduction}

The electric generator is a critical plant item in a power station. During its life time the generator is subject to several kinds of stress, which are generally categorized as thermal, electrical, ambient or mechanical in nature. These stresses affect the reliability of the machine and contribute to the risk of a costly forced outage. The maintenance regime of the generator typically involves periodic disassembly, inspection of critical components, and rehabilitative works as necessary.

This paper introduces two new technologies for inspecting the generator while the generator rotor remains in place within the stator. Both systems are designed for two pole turbo generator types with a cylindrical rotor. The new technologies discussed here leverage robotics to facilitate a rapid inspection of the generator, while avoiding the time and costs associated with rotor removal. Such inspections can be performed between major overhauls in order to increase machine condition information and thereby increase reliability, or alternatively, such inspections could be used to justify prolonging the time between major overhauls and to optimize the outage planning.

This paper is organized as follows. The first part of the paper is used to describe a new robotic system for 
performing inspections within the air gap between generator rotor and stator. While the second part of the paper is used to describe a new robotic system for performing ultrasonic inspections of the generator retaining rings. An overview of the design and construction of each robotic system is provided, along with an explanation of the capability of each system and its associated benefits to the power station owner. The paper also contains several photos to depict the functionality of each technology within a real world context.

\section{Robotic Inspection with Rotor in-Situ}

\subsection{Inspection within the Generator Air Gap}

The company Alstom has been involved in the development of robotic tooling to access and inspect within the air gap between generator stator and rotor for several decades. The original tooling base was geared towards large hydrogen cooled generators [1] [2]. The latest generation system to be introduced to the power market is known as DIRIS ${ }^{\circledR}$ Small, where the acronym DIRIS is derived from the expression "Diagnostic Inspection with Rotor In-situ”. This latest generation robotic system is highlighted in this paper as a novel device due to its ability to perform inspections within very narrow air gaps typically found on small air cooled generators with challenging geometrical constraints.

The DIRIS Small robot depicted in Figure 1 is designed such that it is positioned on one generator retaining ring and has computer controlled axial and circumferential movement to facilitate the inspection process. Once positioned circumferentially, the axial probe carrier can extend along the length of each stator slot section. This process can be repeated a full 360 degrees around the stator bore. Typically one human operator can control the entire process from outside the generator using proprietary software, while another human operator periodically checks the position of the tooling for any unforeseen obstructions and acts as a secondary observer of mechanical quality assurance.

The DIRIS Small robot is capable of performing critical tests of the generator stator iron core laminations, stator radial wedging system and conducting a visual (video type) inspection of the inside surfaces of the rotor and stator [1]. These tests would normally be part of a typical overhaul regime after a lengthy process of removing the rotor and utilizing manual and semi-automated tooling. The low flux test permits the identification of short circuits between the stator iron core laminations, which could otherwise develop into critical "hot spots" and severely damage the generator [3]. The tightness test of the radial wedging system permits the identification of loose wedges, which could otherwise promote movement of the stator bars and damage to the stator winding insulation system [4].

Generally speaking, the inspection of the air gap using such robotic tooling can be accomplished in less than a week on a day-shift pattern, including the time required to perform mechanical disassembly and reassembly of exterior components. As depicted in Figure 2 and Figure 3 the DIRIS Small robot accesses the air gap between stator and rotor from one side of the machine only. Therefore it follows that the required disassembly of exterior components for an air cooled generator is typically limited to a small amount of pipework, one outer end shield, and an internal air baffle. It should also be noted that experience suggests that in most cases the rotor fan blades are able to remain in place during the inspection.

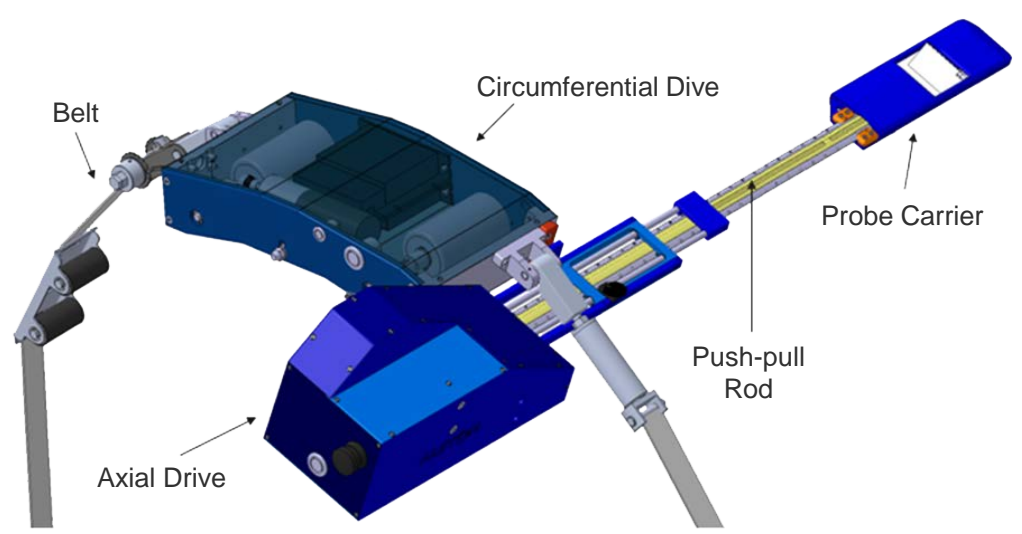

Figure 1. Overview of the DIRIS small air gap robot. 


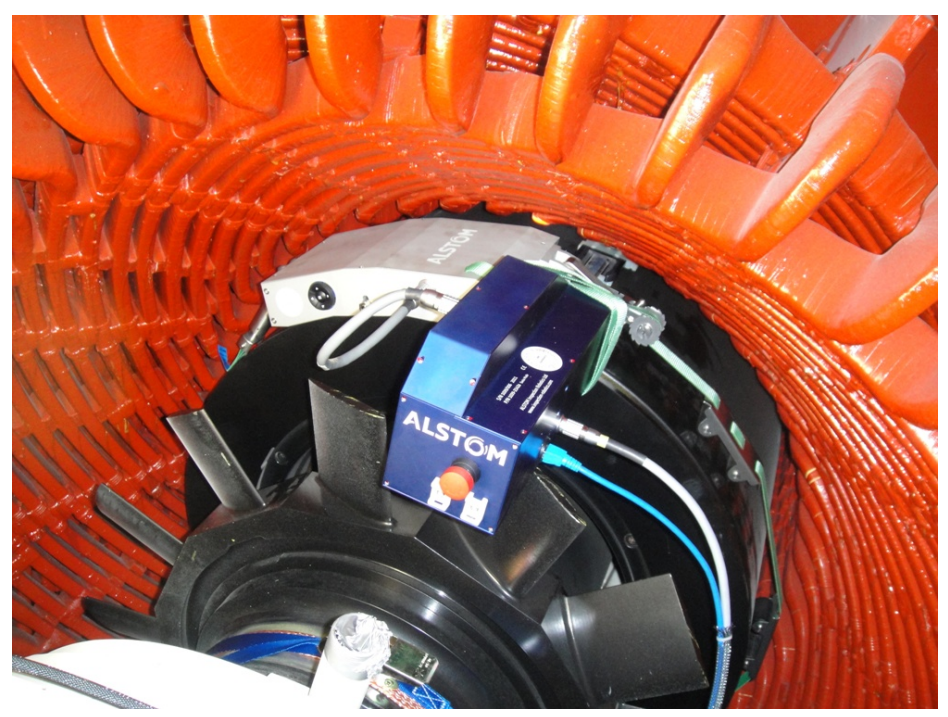

Figure 2 DIRIS Small air gap robot deployed on a 60 MW generator of European manufacturing origin.

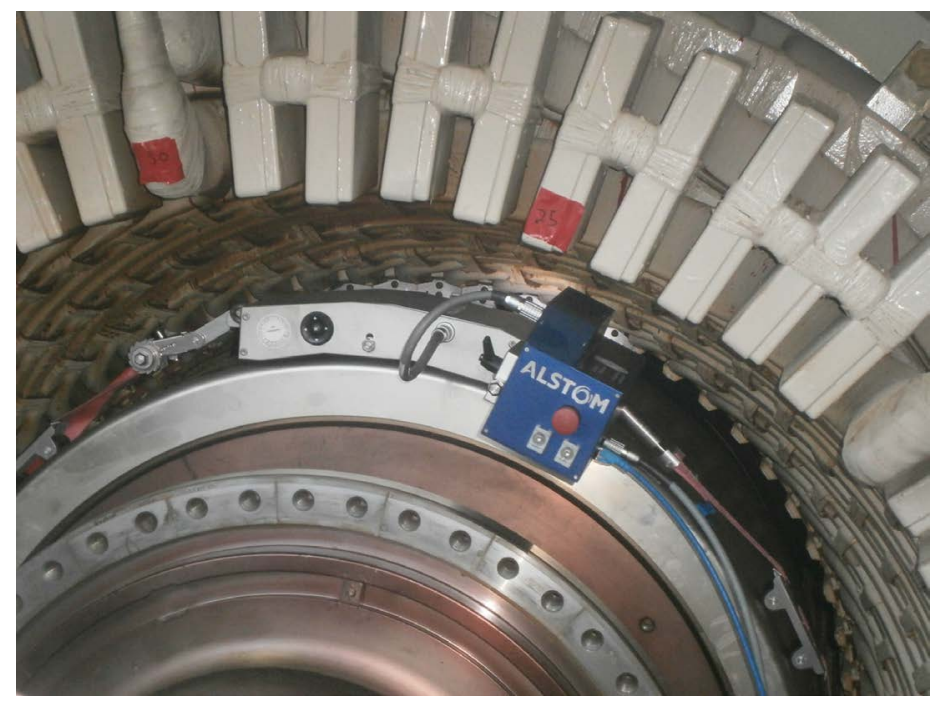

Figure 3DIRIS Small air gap robot deployed on a 175 MW generator of North American manufacturing origin.

\subsection{Inspection of the Generator Retaining Rings}

The retaining rings are a critical component of the generator. In service, the retaining rings provide mechanical support to the generator rotor end windings which are subject to high levels of centrifugal force. The consequences of retaining ring failure have been widely discussed in the literature and for this reason are ignored here (see for example [5]). Historically the retaining rings have been inspected using a dye-penetrant test of the inside surfaces. This process necessitates removal of the generator rotor, followed by removal of the retaining rings using high levels of heat and significant human effort.

The robotic technology described here has also been developed by the company Alstom and provides the flexibility to perform inspections of the generator retaining rings while the rotor remains in place and while the retaining rings are mounted on the rotor. The robotic system for scanning retaining rings introduced in this paper is known as TurboRotoscan-S and depicted in Figure $\mathbf{4}$ and Figure 5. The system is designated by an " $\mathrm{S}$ " (or "Small") as it represents an evolution of an older variant robotic tool intended for large hydrogen cooled generators. The system described here has an extremely lightweight chassis, designed to be mounted directly to the 


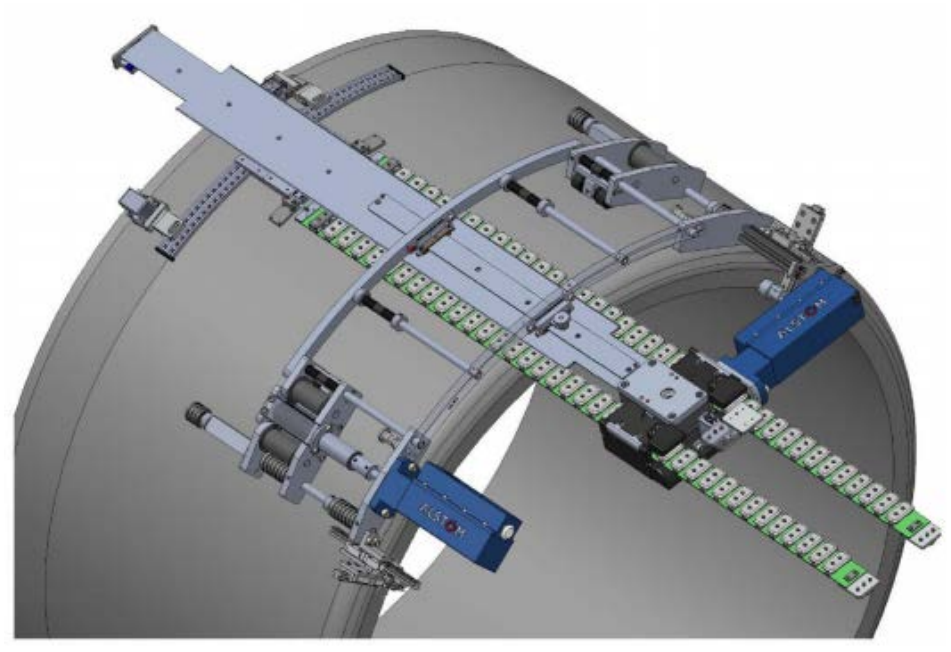

Figure 4. Overview of the TurboRotoscan-S positioned on a simulated generator retaining ring.

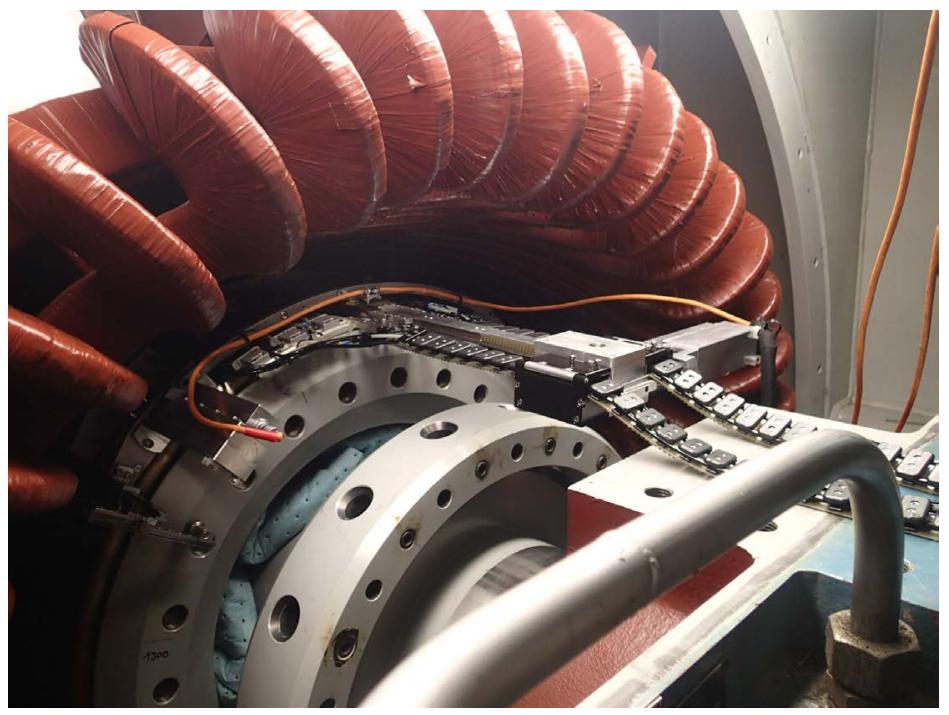

Figure 5. TurboRotoscan-S deployed on a $40 \mathrm{MW}$ generator of European manufacturing origin.

retaining rings and has computer controlled axial and circumferential movement of probe heads to map the body of the retaining ring metal for defects. The system is intended for a wide range of types and sizes of turbo generators. Due to its lightweight structure the robotic tooling can be hand carried to the work site with relative ease.

In its nominal configuration the retaining ring scanner contains on-board probes for phased array ultrasonic inspection, in order to check the inside surfaces of the retaining ring metal for cracks. The scanner also contains an eddy current probe to check the retaining ring outside surfaces. The data acquisition is performed using third party software. In a similar fashion to that described previously, the robotic retaining ring scanner utilizes two human controllers, one to drive the movement from outside the machine and monitor data quality while a secondary human monitors for any unforeseen obstructions inside the end-winding portion of the machine.

Generally speaking, such an inspection of two retaining rings can also be performed in less than a week on a day-shift basis. The required disassembly of exterior components on an air cooled generator is limited to a small amount of pipework, any air guides on the exciter side of the generator, two outer end shields, and two internal air baffles. Depending on the machine design the rotor fan blades may also need to be removed to grant access to the axial rail portion of the scanner. 


\section{Summary Remarks}

Two new robotic technologies to perform fast and reliable inspections of the turbo-generator have been described in brief. Both of these systems are designed for two pole electric generators with cylindrical rotors and bypass the need to remove the generator rotor from the stator. This results in significant savings in mechanical disassembly, reassembly, and downtime costs for the power station owner. The capability of these robotic systems includes low flux stator iron core testing, stator radial wedging assessment, rotor and stator visual inspection, and ultrasonic inspection of the generator retaining rings. Both of these new systems were designed with "real world" constraints in mind, such as consideration for a light weight and slim chassis, transportability, challenging geometrical inspection constraints and a low level of ancillary human labor.

As partially evidenced by the supporting illustrations, both of these new systems have had immediate traction in the power generation market, and have been deployed in a wide variety of generator types. From experience of the authors, it can be stated that both forms of technology have already been deployed on generators of European, North American and Japanese manufacturing origin. The commercial logic for performing inspections with robotic tooling, in place of traditional human means, is not a difficult one to grasp. The benefits of a rapid robotic inspection include the flexibility to assess generator condition in between major overhauls and the ability to make informed decisions to potentially prolong the time between major overhauls.

\section{References}

[1] Sapre, V., Savenkov, M. and Fischer, R. (2014) Advanced Generator Robotic Monitoring—Maximising Availability, Minimising Costs. Proceedings of the 27th International Congress of Condition Monitoring and Diagnostic Engineering, Brisbane, 16-18 September 2014.

[2] Mark, B. (2003) Practical Experiences with Robotic Inspection and Measurement Tools and Their Impact on Maintenance Strategies. Power-Gen Europe, Düsseldorf, 6-8 May 2003.

[3] Posedel, Z. (2001) Inspection of Stator Core for Large Machines with a Low Yoke Induction Method-Measurement and Analysis of Interlamination Short-Circuits. IEEE Transactions on Energy Conversion, 16, 81-86. http://dx.doi.org/10.1109/60.911408

[4] Bloch, M., Baumann, J., Koch, E. and Zimmerli, B. (2010) Alstom Successful Operational Experience with Advanced Stator Bar Wedging System for Turbogenerators. Power-Gen Europe, Amsterdam.

[5] Viswanathan, R. (Ed.) (1982) Workshop Proceedings: Retaining Rings for Electric Generators. Electric Power Research Institute (EPRI) EL-3209 Research Project 1876 Proceedings, Palo Alto, 11-12 October 1982. 